\title{
Risk Factors for Development of Acute Kidney Injury in Critically Ill Patients: A Systematic Review and Meta-Analysis of Observational Studies
}

\author{
Rodrigo Cartin-Ceba, ${ }^{1}$ Markos Kashiouris, ${ }^{1}$ Maria Plataki, ${ }^{1}$ Daryl J. Kor, ${ }^{2}$ \\ Ognjen Gajic, ${ }^{1}$ and Edward T. Casey ${ }^{3}$ \\ ${ }^{1}$ Division of Pulmonary and Critical Care Medicine, Mayo Clinic, 200 First Street SW, Rochester, MN 55905, USA \\ ${ }^{2}$ Department of Anesthesia, Mayo Clinic, 200 First Street SW, Rochester, MN 55905, USA \\ ${ }^{3}$ Division of Nephrology and Hypertension, Mayo Clinic, 200 First Street SW, Rochester, MN 55905, USA
}

Correspondence should be addressed to Rodrigo Cartin-Ceba, cartinceba.rodrigo@mayo.edu

Received 22 June 2012; Accepted 29 August 2012

Academic Editor: Gemma Seller-Pérez

Copyright (c) 2012 Rodrigo Cartin-Ceba et al. This is an open access article distributed under the Creative Commons Attribution License, which permits unrestricted use, distribution, and reproduction in any medium, provided the original work is properly cited.

\begin{abstract}
Background. Acute kidney injury (AKI) is a frequent complication of critically ill patients. The impact of different risk factors associated with this entity in the ICU setting is unknown. Objectives. The purpose of this research was to assess the risk factors associated with the development of AKI in critically ill patients by meta-analyses of observational studies. Data Extraction. Two reviewers independently and in duplicate used a standardized form to collect data from published reports. Authors were contacted for missing data. The Newcastle-Ottawa scale assessed study quality. Data Synthesis. Data from 31 diverse studies that enrolled 504,535 critically ill individuals from a wide variety of ICUs were included. Separate random-effects meta-analyses demonstrated a significantly increased risk of AKI with older age, diabetes, hypertension, higher baseline creatinine, heart failure, sepsis/systemic inflammatory response syndrome, use of nephrotoxic drugs, higher severity of disease scores, use of vasopressors/inotropes, high risk surgery, emergency surgery, use of intra-aortic balloon pump, and longer time in cardiopulmonary bypass pump. Conclusion. The best available evidence suggests an association of AKI with 13 different risk factors in subjects admitted to the ICU. Predictive models for identification of high risk individuals for developing AKI in all types of ICU are required.
\end{abstract}

\section{Introduction}

Acute kidney injury (AKI) is a common and highly lethal problem faced in the intensive care unit (ICU) $[1,2]$, with a reported incidence of 1 to $67 \%$ [3-6], and a mortality that ranges from 28 to $90 \%$ [5, 7-10]. This wide range in incidence and mortality is in part due to the near 35 different definitions of AKI [11]. Different risk factors for the development of AKI in the intensive care unit (ICU) have been assessed in diverse populations, including port-surgical, trauma, and medical patients. A wide variety of risk factors have been described but there is no clear understanding of what risk factors confer the highest risk for development of AKI. In addition, risk factors identified in some studies have not been confirmed in subsequent studies or the effect may differ depending on the clinical setting. Better understanding the impact and the association of different risk factors with AKI is of paramount importance for designing predictive models of high risk patients, and also to create preventive strategies that might benefit patients from developing this lethal condition. Predictive models for development of AKI already exist in cardiac-surgery critically ill patients [1214]; however, refinements are still required to modify these studies into clinically applicable tools, and there is lack of meaningful predictive models in mixed and medical ICUs where most of the prediction models have focused on the impact on mortality of AKI in ICU patients [15, 16]. Given this situation, how can we identify patients at risk for AKI and are there interventions to mitigate this risk? To answer the former question in order to be able to respond the latter one, we set out to conduct a systematic review of the risk factors associated with the development of AKI in the ICU. 


\section{Methods}

The report of this protocol-driven systematic review and meta-analysis adheres to the Preferred Reporting Items for Systematic Reviews and Meta-Analyses (PRISMA) standards for reporting systematic reviews and meta-analysis studies [17].

2.1. Study Eligibility. Eligible studies were observational studies (prospective and retrospective cohorts or casecontrol studies) where specific risk factors for AKI were investigated in critically ill adult $(\geq 18)$ patients with no AKI at the time of ICU admission. Studies were required to have a control group with no AKI for comparison and the main outcome of interest was development of AKI by any definition during the ICU stay (definition must include criteria based on change in creatinine levels). Study inclusion was not limited by publication status or language. Exclusion criteria included studies assessing specific causes of AKI such as rapidly progressive glomerulonephritis, hemolytic-uremic syndrome, or hepatorenal syndrome, and also specialized ICUs (burn and transplant ICUs). Studies must have excluded end stage kidney disease patients.

2.2. Search Strategy. An expert medical librarian with extensive meta-analytical experience collaborated to design the search strategies. The following databases were searched since inception of the database until the third week of January of 2010: Ovid MEDLINE, Ovid EMBASE, Cochrane Library, Web of Science, and Scopus. Database-specific controlled language and terms that describe the key concepts were utilized: acute kidney injury, acute renal failure, acute renal insufficiency, risk factors, intensive care unit, critically ill, outcomes, and observational studies. The detailed search strategy is described in Appendix 1, in supplementary material available online at doi:10.1155/2012/691013. We also reviewed the reference sections of identified reviews, published guidelines, and published manuscripts known to the authors. In addition, we reviewed the reference sections of eligible studies.

2.3. Study Selection. Using a high threshold for exclusion, pairs of reviewers, working independently and in duplicate, identified potentially eligible studies for full-text retrieval from the titles and abstracts. Studies in which the reviewers disagreed were also retrieved in full text. Subsequently, disagreements were harmonized by consensus; when this was not possible, an independent reviewer reviewed and resolved the discordance by arbitration. We assessed interobserver agreement by the kappa statistics. Two independent native French speakers reviewed 2 studies published in French; similarly, 2 independent native Spanish speakers reviewed 2 studies published in Spanish.

2.4. Data Extraction. Two investigators independently used standardized forms to abstract descriptive, methodological, and outcome data from all eligible studies. The reviewers utilized a standardized data extraction form that include characteristics of the individual studies (sample size, gender distribution), clinical setting (surgical (trauma, cardiac, or general), mixed or medical ICU) and country, number of centers involved in the study, type of study (prospective or historical cohort, case-control study), years of enrollment, definition of AKI utilized, incidence of AKI in the study, and risk factors for AKI. Because of significant inconsistency in reporting risk factors among the studies, we assessed the following risk factors deemed to be of significant importance in the developing of AKI in all the eligible studies: age, diabetes, hypertension, heart failure, baseline serum creatinine (as a measure of evidence of chronic kidney disease), sepsis/systemic inflammatory response syndrome (SIRS), nephrotoxic drugs (including intravenous contrast dye, aminoglycosides, amphotericin $\mathrm{B}$, vancomycin, nonsteroidal anti-inflammatory drugs, angiotensin converting enzyme inhibitors, and angiotensin receptor blockers), severity of disease as measured by different severity scores such as the acute physiologic and chronic health evaluation (APACHE) $[18,19]$ or the injury severity score (ISS) [20], hypotension or shock, use of vasopressors and/or inotropes, high risk surgery, and emergency surgery. High risk surgery and emergency surgery were not assessed in medical ICUs for obvious reasons. For cardiosurgical ICUs, we also assessed the use of intra-aortic balloon pump (IABP) and the time on the cardiopulmonary bypass pump in minutes.

2.5. Quality Assessment. Independent reviewers working in duplicate determined the quality of each study based on the Newcastle-Ottawa quality assessment scale (NOS) for cohorts and case control studies [21]. Disagreements were harmonized by consensus. When this was not possible, a third reviewer adjudicated the quality of the questionable study.

2.6. Statistical Analysis. The main outcome of assessment was the development of AKI. The risk estimate analyzed for every risk factor in the studies was the odds ratio (OR) for dichotomous variables, and the mean difference for the continuous variables with its corresponding $95 \%$ confidence interval (CI). When available, we used the adjusted risk estimates (adjusted OR) from multivariate models. For severity of disease, because of diverse severity of disease scoring systems, we estimated the point estimate for each study using the effect size (ES), which expresses the effect of the risk factor in terms of the standard deviation of the measurement producing a unitless estimate that can be compared across studies [22]. We performed separate meta-analyses with the random effects model [23] to obtain the pooled OR or pooled mean difference for the development of AKI for each risk factor with its corresponding 95\% CI. We then used the $I^{2}$ statistic to quantify the proportion of observed inconsistency across study results not explained by chance [24]. $I^{2}$ values of less than $25 \%, 50 \%$, and more than $75 \%$ represent low, moderate, and high inconsistency, respectively [24]. We proposed predefined subgroup analyses based on our a priori hypotheses to explain potential heterogeneity across studies on the strength of association due to different 
ICU types (surgical, mixed, and medical), considering a significant interaction when $P<0.05$. In addition, in order to evaluate the impact of studies with poor methodological quality, we conducted sensitivity analyses excluding studies in the lower 2 quartiles of the NOS quality assessment scale scores. Comparisons of risk estimates between subgroups and sensitivity analyses were made with a test of interaction [25]. The presence of publication bias was investigated graphically by the method of Sterne and Egger [26]. All analyses were performed with Review Manager Software (RevMan Analyses Version 5.0.4 Copenhagen; The Nordic Cochrane Center, The Cochrane Collaboration, 2008).

\section{Results}

3.1. Search Results and Study Inclusion. Our initial search identified 829 unique publications in the form of titles and abstracts, which were narrowed by preliminary review to 108 potentially relevant original articles that were examined in full text. The interobserver agreement in this phase was $\kappa=0.90$ (95\% CI, 0.88-0.93). The search of references from the 108 retrieved papers identified 3 additional articles. Figure 1 describes the flow diagram of the process of study selection and the reasons for exclusion of studies. Ultimately, our systematic review and meta-analysis included 31 studies $[4-6,27-54]$ that evaluated a total of 504,535 individuals.

Eleven studies were prospective cohorts, sixteen studies were historical cohorts, three studies were case-control studies, and one study was a nested case-control study. Most of these studies were done in surgical ICUs $(n=20 ; 14$ of which were cardiothoracic ICUs); the remainder were mixed ICUs $(n=8)$, and medical ICUs $(n=3)$. There were a wide range of years of enrollment of the cohorts, from 1976 to as recent as 2008. A total of 26 different definitions of AKI were identified in these 31 studies, all had in common a prespecified elevation of creatinine or an elevation of creatinine from baseline. The incidence of AKI in the different ICUs as defined by the multiple definitions ranged from 1.2 to $67 \%$. Table 1 describes the studies' characteristics in detail.

3.2. Methodological Quality. Table 2 summarizes the quality of the studies utilizing the NOS quality assessment scale. The median score of the studies was 6 (interquartile range, 6 to 8 ), where most of the studies failed to demonstrate that the outcome (AKI) was not present at start of the study. Moreover, only 14 studies performed a multivariate analysis for adjustment of baseline imbalances and confounders.

3.3. Meta-Analyses. Separate meta-analyses for each risk factor demonstrated that all assessed risk factors, with the exception of hypertension, were significantly associated with the development of AKI in critically ill patients as depicted in Table 3. There was a trend of association of hypertension with AKI; however, it was not statistically significant (OR $1.15,95 \%$ CI $0.76,1.74$ ). After excluding 4 studies that assessed hypertension due to poor quality (see sensitivity analyses), no interaction was observed but the risk estimate was significant (OR $1.4395 \%$ CI 1.08, 1.89), and there was also an improvement in heterogeneity between studies. Higher levels of creatinine at baseline and a higher severity of disease score (equivalent difference of 18 points in the APACHE III score, 9 points in the ISS, and 6 points in the APACHE II score) were also associated with AKI. Besides these associations, patients in cardiothoracic ICUs that developed AKI showed a significant association with the use of IABP (OR 3.29, 95\% CI 2.21, 4.91) and with longer time on the cardiopulmonary bypass pump (mean difference 27.92, 95\% CI 14.41, 41.43 minutes). Table 3 also describes the reporting of the different risks appraised. It is noticeable that with the exemption of age, "reporting bias" is very suggestive in the remainder risk factors. Very few risk factors were also reported as part of multivariate adjustment and, when available, these results were included in the analyses. Significant heterogeneity existed between studies among the different risk factors evaluated $\left(I^{2}>75 \%\right)$, with the only exemption of nephrotoxic drugs that presented moderate heterogeneity with an $I^{2}$ of $42 \%$.

3.4. Subgroup Analyses. Preplanned subgroup analyses were performed for every risk factor according to the type of ICU (surgical, mixed, or medical). Table 4 demonstrates that significant interaction was only observed in diabetes and heart failure between mixed ICUs, and high risk/emergency surgery in surgical and mixed ICUs. The point estimate association of diabetes and heart failure in the development of AKI in mixed ICUs remained significant but lower than the pooled estimate. Patient characteristics' differences regarding the inclusion of both medical and surgical patients in the mixed ICUs might account for the observed discrepancy, and less likely the studies included in the subgroup analyses which presented low heterogeneity for both diabetes $\left(I^{2} 0 \%\right)$ $[34,49,50,53]$ and heart failure $\left(I^{2} 0 \%\right)[4,34,50,53]$. Furthermore, outcomes of patients that develop AKI in surgical ICUs tend to be worse than in medical ICUs [9].

The effect of high risk/emergency surgery between surgical and mixed ICUs likely represents opposite effects of the two types of ICUs in the pooled point estimate. The estimate of association with AKI is not significant for high risk/emergency surgery in mixed ICUs as opposed to surgical ICUs, where the point estimate is even higher. A possible explanation could be that the risk is likely diluted due to the inclusion of both "surgical" and "medical" patients in mixed ICUs. To confirm this hypothesis, we excluded the study with higher reported number of medical patients as opposed to surgical patients in the mixed with ICUs [4] and the resulted OR was 1.52 (95\% CI 1.10, 2.09), with a test for interaction that was not significant $(P=0.09)$.

3.5. Sensitivity Analyses. We performed sensitivity analyses to test how robust the results of the meta-analyses were in relation to the methodological quality of the studies. Studies with a NOS score $<6$ were excluded in our sensitivity analyses. Our results were not significantly altered by the exclusion of studies with poor methodological quality, with mild inconsistency improvement, particularly in diabetes, 


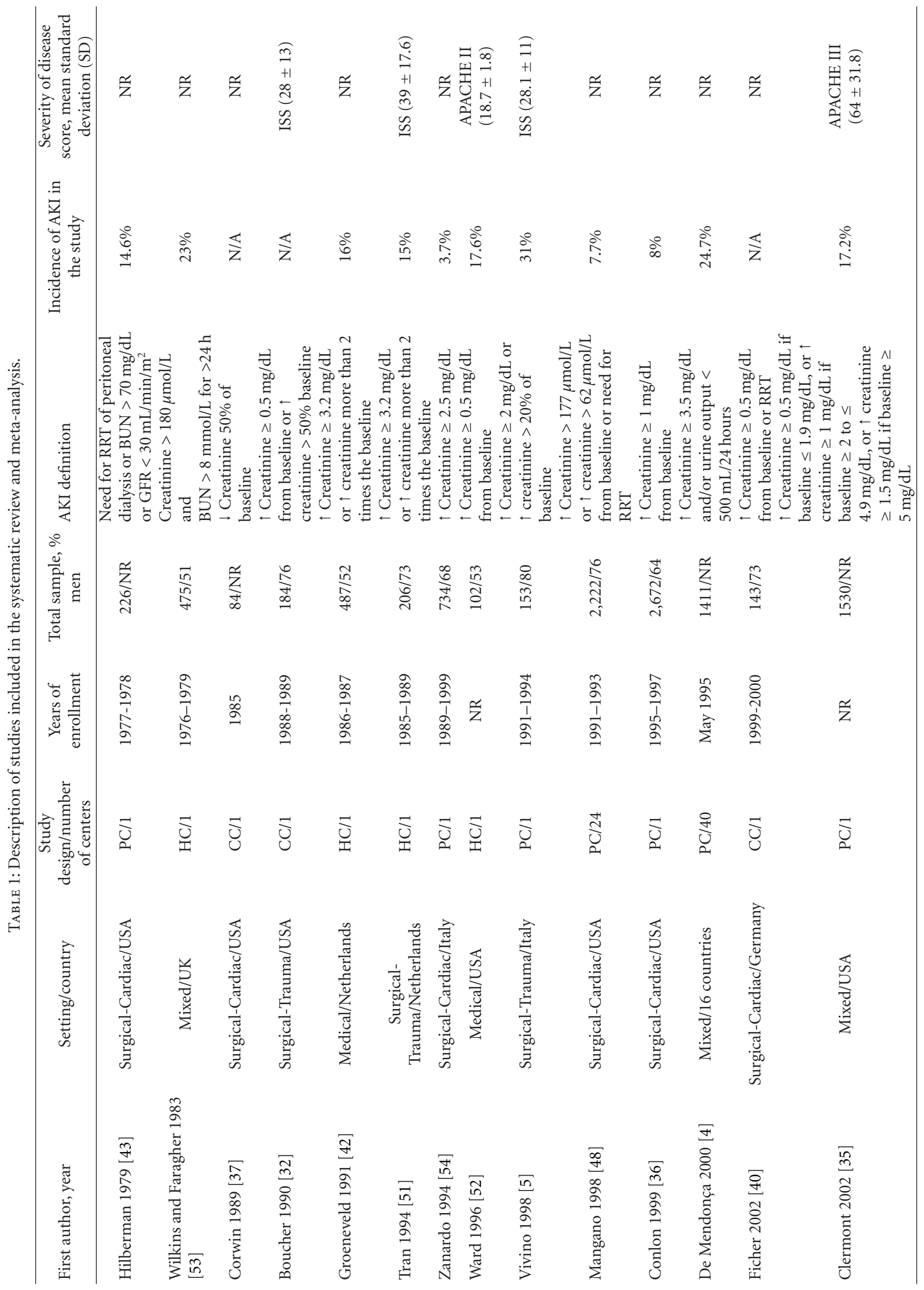




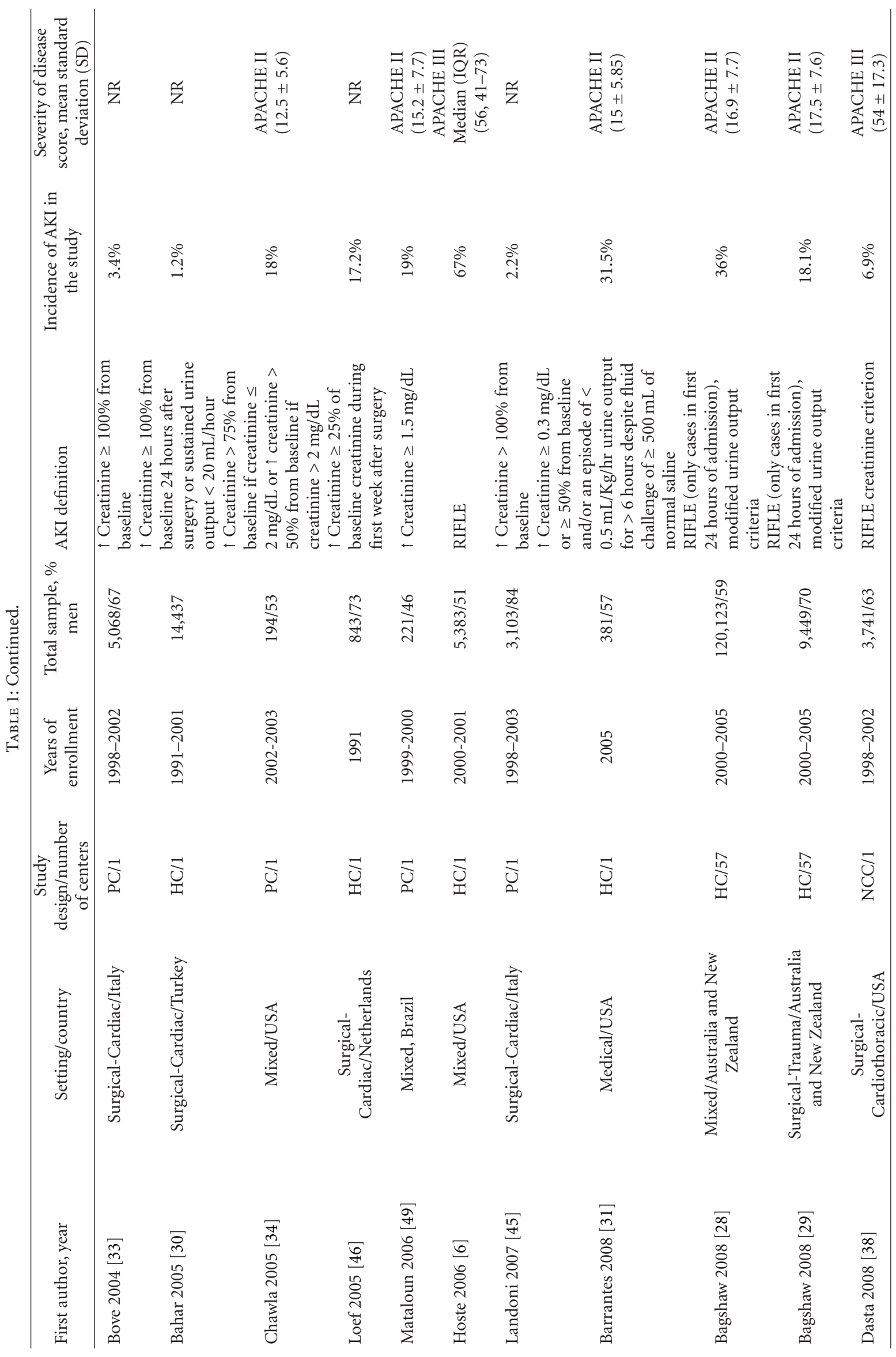




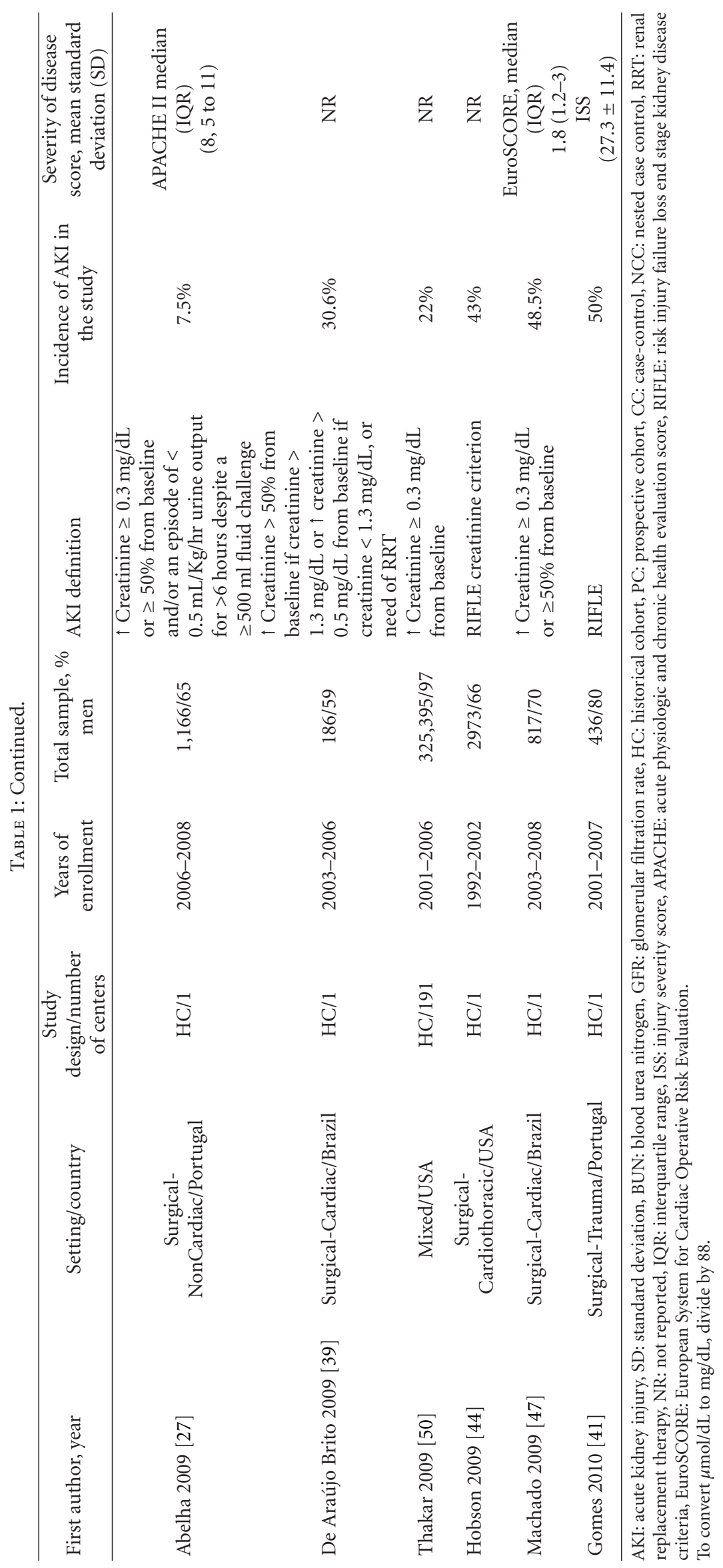




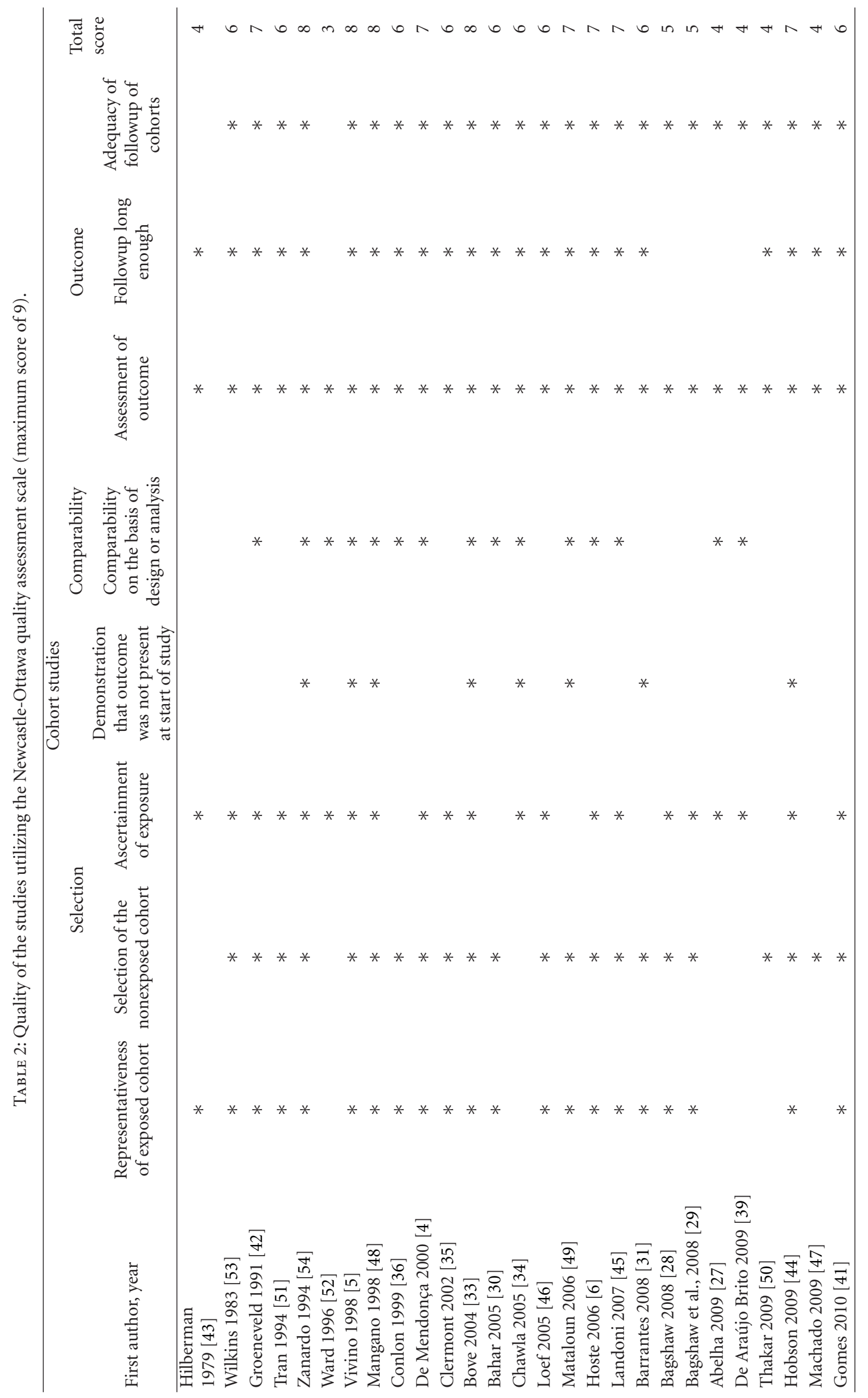




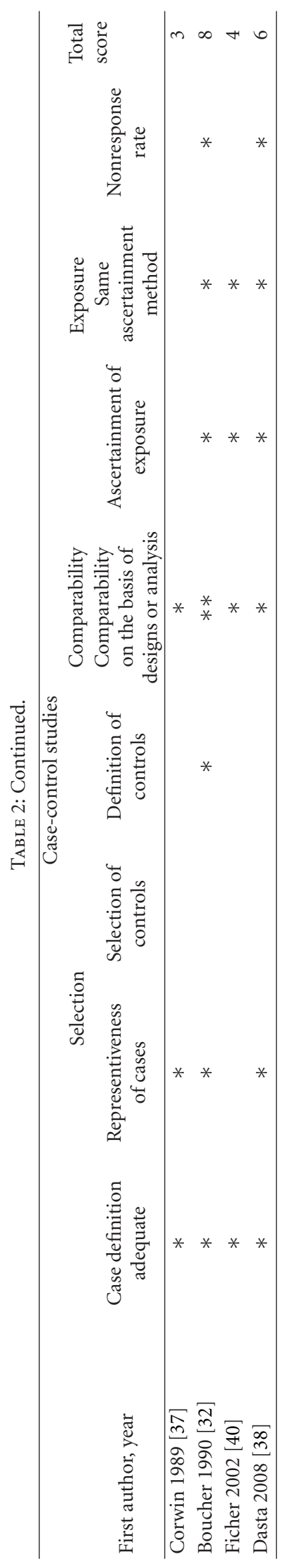




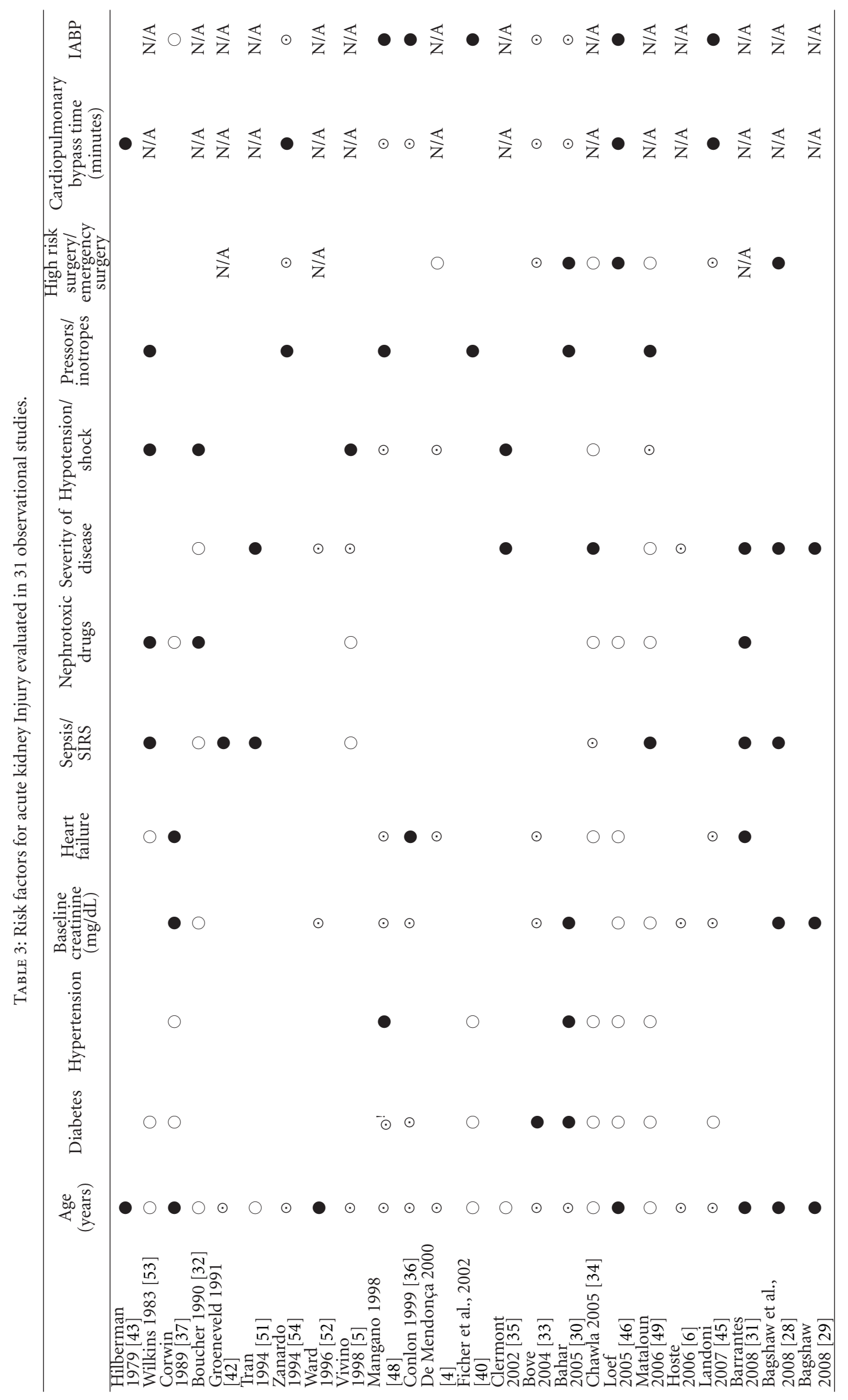




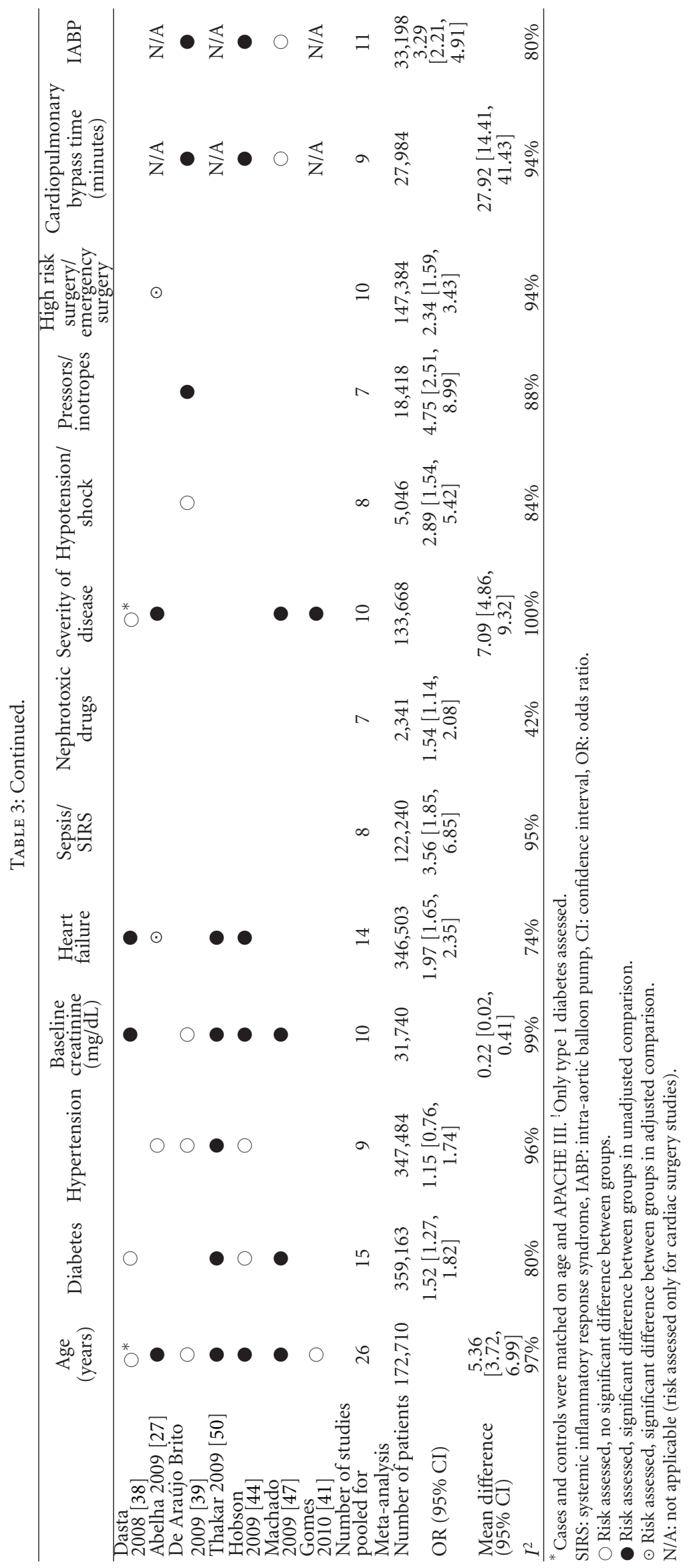


TABLE 4: Subgroups analysis.

\begin{tabular}{|c|c|c|c|c|}
\hline Risk Factor & Number of studies & 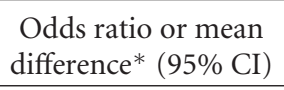 & $\begin{array}{l}P \text { value for } \\
\text { interaction }\end{array}$ & $\begin{array}{c}\text { Inconsistency } \\
I^{2}\end{array}$ \\
\hline \multicolumn{5}{|l|}{ Age } \\
\hline Surgical & 17 & $5.35[2.84,7.86]$ & 0.99 & $96 \%$ \\
\hline Mixed & 6 & $4.95[2.27-7.63]$ & 0.81 & $97 \%$ \\
\hline Medical & 3 & $7.61[4.77,10.44]$ & 0.17 & $0 \%$ \\
\hline \multicolumn{5}{|l|}{ Diabetes } \\
\hline Surgical & 11 & $1.61[1.41,1.83]$ & 0.61 & $31 \%$ \\
\hline Mixed & 4 & $1.09[1.07,1.11]$ & $<0.001$ & $0 \%$ \\
\hline \multicolumn{5}{|c|}{ Hypertension } \\
\hline Surgical & 7 & $1.34[1.04,1.73]$ & 0.53 & $68 \%$ \\
\hline Mixed & 2 & $0.68[0.45,1.05]$ & 0.08 & $49 \%$ \\
\hline \multicolumn{5}{|c|}{ Baseline creatinine* } \\
\hline Surgical & 8 & $0.20[-0.02,0.42]$ & 0.89 & $99 \%$ \\
\hline \multicolumn{5}{|c|}{ Heart Failure } \\
\hline Surgical & 9 & $2.19[1.92,2.5]$ & 0.34 & $8 \%$ \\
\hline Mixed & 4 & $1.51[1.47,1.55]$ & 0.003 & $0 \%$ \\
\hline \multicolumn{5}{|l|}{ Sepsis/SIRS } \\
\hline Surgical & 2 & $2.69[1.28,5.64]$ & 0.57 & $47 \%$ \\
\hline Mixed & 4 & $2.52[1.36,4.69]$ & 0.45 & $87 \%$ \\
\hline Medical & 2 & $8.58[1.56,47.22]$ & 0.34 & $95 \%$ \\
\hline \multicolumn{5}{|c|}{ Nephrotoxic drugs } \\
\hline Surgical & 4 & $1.25[0.92,1.71]$ & 0.34 & $0 \%$ \\
\hline Mixed & 2 & $2.12[1.04,4.32]$ & 0.41 & $64 \%$ \\
\hline \multicolumn{5}{|c|}{ Severity of disease* } \\
\hline Surgical & 5 & $5.69[3.25,8.14]$ & 0.40 & $88 \%$ \\
\hline Mixed & 3 & $10.66[3.37,17.95]$ & 0.35 & $96 \%$ \\
\hline Medical & 2 & $5.32[3.98,6.65]$ & 0.18 & $0 \%$ \\
\hline \multicolumn{5}{|c|}{ Hypotension/shock } \\
\hline Surgical & 4 & $2.48[1.3,4.72]$ & 0.74 & $57 \%$ \\
\hline Mixed & 4 & $3.44[1.09,10.89]$ & 0.79 & $92 \%$ \\
\hline \multicolumn{5}{|c|}{ Pressors/inotropes } \\
\hline Surgical & 5 & $5.36[2.39,12.03]$ & 0.81 & $91 \%$ \\
\hline Mixed & 2 & $3.41[1.87,6.20]$ & 0.45 & $19 \%$ \\
\hline \multicolumn{5}{|c|}{$\begin{array}{l}\text { High risk surgery/emergency } \\
\text { surgery }\end{array}$} \\
\hline Surgical & 6 & $3.79[2.91,4.94]$ & 0.04 & $33 \%$ \\
\hline Mixed & 4 & $1.14[0.64,2.02]$ & 0.04 & $91 \%$ \\
\hline
\end{tabular}

CI: confidence interval, SIRS: systemic inflammatory response syndrome. 


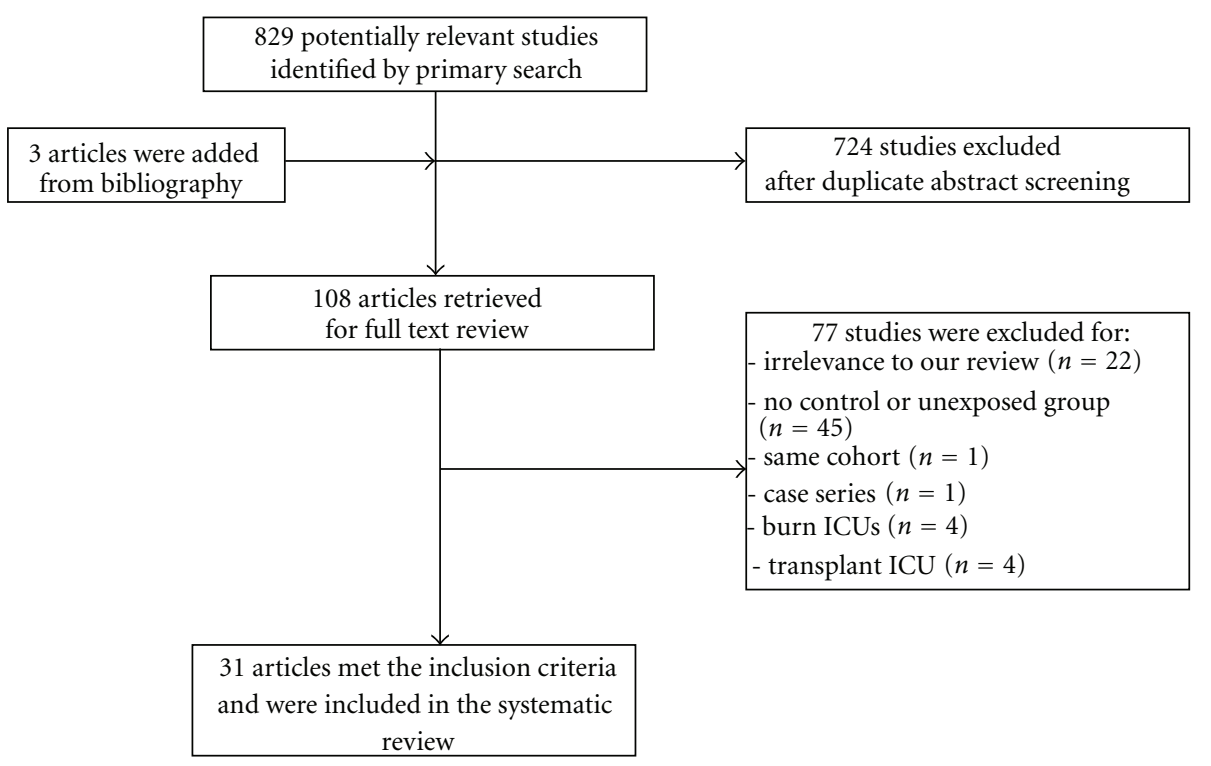

FIgURE 1: Flow diagram of the process of study selection.

heart failure, nephrotoxic drugs, and cardiopulmonary bypass time as described in Table 5 .

3.6. Publication Bias. The funnel plots for every individual risk factor are presented in Appendix 2, in supplementary material available online at doi:10.1155/2012/691013. Most of the plots showed asymmetry, suggesting small-study bias (either the absence of or inability to find studies with smaller or negative risk estimates) or unexplained heterogeneity.

\section{Discussion}

This study found that the current evidence, drawn from a large number of observational studies that included more than half million individuals, indicates a significantly increased risk of AKI in critically ill patients with older age, diabetes, higher baseline creatinine, heart failure, sepsis/SIRS, use of nephrotoxic drugs, higher severity of disease scores, use of vasopressors/inotropes, high risk surgery, emergency surgery, and possibly hypertension. Additionally, cardiothoracic patients admitted to the ICU also presented increased risk of AKI with the use of IABP and longer time in cardiopulmonary bypass pump. We found that many of these observational studies were methodologically limited and presented high levels of heterogeneity. Multiple definitions of AKI, differences in populations studied, differences in the internal characteristics and practice of the diverse ICUs, differences in countries, and differences in processes of care over time might explain the inconsistencies identified in this review. Most of the definitions of AKI utilized in these studies possess high specificity for the diagnosis of AKI but poor sensitivity due to lack of urine output criteria [55]. With the exemptions already described, the risk factors described in this review that were associated with AKI presented similar point estimates across different types of ICUs. It was reassuring that after exclusion of methodological limited studies, all point estimates remained significant. Our findings demonstrated important methodological issues observed in several of the studies analyzed, particularly the potential impact of "reporting bias" and lack of adjustment for important covariates in observational studies.

4.1. Limitations/Strengths. Our results are limited to the extent that the observational studies included in this systematic review yielded inconsistent and imprecise data about association effects. One might argue the legitimacy of combining dissimilar patients from different types of ICUs. Our analysis was subject to the limitation of different definitions of AKI among the studies (Table 1), which in fact may have contributed to the unexplained heterogeneity that we have identified between our selected studies. This differential approach in defining AKI could have potentially overestimated or underestimated some the risk factors identified in our analysis. However, the subgroup analysis looking at each type of ICU did not result in significantly different point estimates for the vast majority of the risk factors. Furthermore, in all systematic reviews, the usual key limitation is publication and reporting bias. Our review clearly showed evidence of significant reporting bias and possible publication bias. The strengths of this systematic review stem from the exhaustive literature search, a thorough evaluation of the methodological quality of the studies, and a focused analysis with complete, prespecified subgroup, and sensitivity analyses. Furthermore, the most compelling evidence comes from our pooled analysis of studies that included thousands of patients. Finally, our contact of the study authors to obtain missing data and their collaboration with our request further strengthen the quality of the review.

4.2. Clinical Implications and Future Research. Our findings should be considered for designing prediction models of AKI 
TABLE 5: Sensitivity analysis.

\begin{tabular}{|c|c|c|c|c|}
\hline Risk factor & $\begin{array}{c}\text { Number of studies } \\
\text { excluded }\end{array}$ & $\begin{array}{l}\text { Odds ratio or mean } \\
\text { difference* }(95 \% \mathrm{CI})\end{array}$ & $\begin{array}{l}P \text { value for } \\
\text { interaction }\end{array}$ & Inconsistency $I^{2}$ \\
\hline Age & 9 & $4.95[3.79,6.12]$ & 0.69 & $80 \%$ \\
\hline Diabetes & 4 & $1.58[1.36,1.84]$ & 0.74 & $36 \%$ \\
\hline Hypertension & 4 & $1.43[1.08,1.89]$ & 0.39 & $73 \%$ \\
\hline Baseline creatinine* & 3 & $0.14[0.01,0.27]$ & 0.50 & $94 \%$ \\
\hline Heart failure & 2 & $2.05[1.77,2.38]$ & 0.73 & $27 \%$ \\
\hline Sepsis/SIRS & 1 & $4.15[2.36,7.32]$ & 0.72 & $83 \%$ \\
\hline Nephrotoxic drugs & 1 & $1.53[1.09,2.14]$ & 0.97 & $52 \%$ \\
\hline Severity of disease* & 4 & $9.08[4.57,13.60]$ & 0.43 & $94 \%$ \\
\hline Hypotension/shock & 1 & $3.33[1.70,6.52]$ & 0.76 & $84 \%$ \\
\hline Pressors/inotropes & 2 & $4.52[2.03,10.05]$ & 0.92 & $92 \%$ \\
\hline $\begin{array}{l}\text { High risk surgery/emergency } \\
\text { surgery }\end{array}$ & 2 & $2.34[1.23,4.49]$ & 0.99 & $92 \%$ \\
\hline Cardiopulmonary bypass time* & 3 & $30.46[23.41,37.51]$ & 0.74 & $59 \%$ \\
\hline IABP & 3 & $3.76[2.54,5.57]$ & 0.64 & $78 \%$ \\
\hline
\end{tabular}

CI: confidence interval, SIRS: Systemic inflammatory response syndrome, IABP: intra-aortic balloon pump.

in different ICUs with the overarching goal of implementing strategies to prevent this highly lethal and morbid condition. The results provided in this review are also applicable to clinical practice and counseling of patients at high risk of development of AKI. From the research perspective, the potential role of diabetes and heart failure in critically ill AKI patients from different ICU types warrant further investigation.

\section{Conclusions}

While meta-analyses of observational studies carry significant limitations inherent to the design of the studies, this review assessed and confirmed the association of 13 different risk factors in the development of AKI in critically ill adults from 31 studies of more than half million patients. This review demonstrated a significantly increased risk of AKI in critically ill patients with older age, diabetes, hypertension, higher baseline creatinine, heart failure, sepsis/SIRS, use of nephrotoxic drugs, higher severity of disease scores, use of vasopressors/inotropes, high risk surgery, emergency surgery, use of IABP, and longer time in cardiopulmonary bypass pump. Early identification of patients at risk of AKI may help to implement interventions to mitigate this highly lethal condition.

\section{List of Abbreviations}

AKI: $\quad$ Acute kidney injury

APACHE: Acute physiologic and chronic health evaluation

BUN: Blood urea nitrogen

CC: $\quad$ Case control study

CI: $\quad$ Confidence interval

ES: $\quad$ Effect size

GFR: Glomerular filtration rate
EuroSCORE: European System for Cardiac Operative Risk Evaluation

HC: $\quad$ Historical cohort study

IABP: Intra-aortic balloon pump

ICU: Intensive care unit

IQR: Interquantile range

ISS: $\quad$ Injury severity score

N/A: $\quad$ Not applicable

NCC: $\quad$ Nested case control study

NOS: $\quad$ Newcastle-Ottawa scale

NR: $\quad$ Not reported

OR: $\quad$ Odds ratio

PC: $\quad$ Prospective cohort study

PRISMA: Preferred Reporting Items for Systematic Reviews and Meta-Analyses

RIFLE: Risk injury failure loss end stage kidney disease criteria

RRT: $\quad$ Renal replacement therapy

SD: $\quad$ Standard deviation

SIRS: $\quad$ Systemic inflammatory response syndrome.

\section{Acknowledgments}

The authors thank the medical librarian Patricia J. Erwin for her assistance in performing the search for studies. They also thank Victor Montori for his comments and suggestions.

\section{References}

[1] S. Uchino, J. A. Kellum, R. Bellomo et al., "Acute renal failure in critically ill patients: a multinational, multicenter study," Journal of the American Medical Association, vol. 294, no. 7, pp. 813-818, 2005.

[2] S. Uchino, "The epidemiology of acute renal failure in the world," Current Opinion in Critical Care, vol. 12, no. 6, pp. 538-543, 2006. 
[3] G. M. Chertow, S. H. Soroko, E. P. Paganini et al., "Mortality after acute renal failure: models for prognostic stratification and risk adjustment," Kidney International, vol. 70, no. 6, pp. 1120-1126, 2006.

[4] A. De Mendonça, J. L. Vincent, P. M. Suter et al., "Acute renal failure in the ICU: risk factors and outcome evaluated by the SOFA score," Intensive Care Medicine, vol. 26, no. 7, pp. 915$921,2000$.

[5] G. Vivino, M. Antonelli, M. L. Moro et al., "Risk factors for acute renal failure in trauma patients," Intensive Care Medicine, vol. 24, no. 8, pp. 808-814, 1998.

[6] E. A. J. Hoste, G. Clermont, A. Kersten et al., "RIFLE criteria for acute kidney injury are associated with hospital mortality in critically ill patients: a cohort analysis," Critical Care, vol. 10, no. 3, article R73, 2006.

[7] F. Cosentino, C. Chaff, and M. Piedmonte, "Risk factors influencing survival in ICU acute renal failure," Nephrology Dialysis Transplantation, vol. 9, supplement 4, pp. 179-182, 1994.

[8] P. G. H. Metnitz, C. G. Krenn, H. Steltzer et al., "Effect of acute renal failure requiring renal replacement therapy on outcome in critically ill patients," Critical Care Medicine, vol. 30, no. 9, pp. 2051-2058, 2002.

[9] Z. Ricci, D. Cruz, and C. Ronco, "The RIFLE criteria and mortality in acute kidney injury: a systematic review," Kidney International, vol. 73, no. 5, pp. 538-546, 2008.

[10] H. Schiffl, S. M. Lang, and R. Fischer, "Daily hemodialysis and the outcome of acute renal failure," New England Journal of Medicine, vol. 346, no. 5, pp. 305-310, 2002.

[11] J. A. Kellum, "The acute dialysis quality initiative: methodology," Advances in Renal Replacement Therapy, vol. 9, no. 4, pp. 245-247, 2002.

[12] E. B. Fortescue, D. W. Bates, and G. M. Chertow, "Predicting acute renal failure after coronary bypass surgery: cross- validation of two risk-stratification algorithms," Kidney International, vol. 57, no. 6, pp. 2594-2602, 2000.

[13] B. O. Eriksen, K. R. S. Hoff, and S. Solberg, "Prediction of acute renal failure after cardiac surgery: retrospective cross-validation of a clinical algorithm," Nephrology Dialysis Transplantation, vol. 18, no. 1, pp. 77-81, 2003.

[14] R. H. Mehta, J. D. Grab, S. M. O’Brien et al., "Bedside tool for predicting the risk of postoperative dialysis in patients undergoing cardiac surgery," Circulation, vol. 114, no. 21, pp. 2208-2216, 2006.

[15] N. Y. Abosaif, Y. A. Tolba, M. Heap, J. Russell, and A. M. E. Nahas, "The outcome of acute renal failure in the intensive care unit according to RIFLE: model application, sensitivity, and predictability," American Journal of Kidney Diseases, vol. 46, no. 6, pp. 1038-1048, 2005.

[16] N. V. Kolhe, P. E. Stevens, A. V. Crowe, G. W. Lipkin, and D. A. Harrison, "Case mix, outcome and activity for patients with severe acute kidney injury during the first 24 hours after admission to an adult, general critical care unit: application of predictive models from a secondary analysis of the ICNARC Case Mix Programme Database," Critical Care, vol. 12, supplement 1, p. S2, 2008.

[17] D. Moher, A. Liberati, J. Tetzlaff, and D. G. Altman, "Preferred reporting items for systematic reviews and meta-analyses: the PRISMA statement," Annals of Internal Medicine, vol. 151, no. 4, pp. 264-269, 2009.

[18] W. A. Knaus, E. A. Draper, D. P. Wagner, and J. E. Zimmerman, "APACHE II: a severity of disease classification system," Critical Care Medicine, vol. 13, no. 10, pp. 818-829, 1985.
[19] W. Knaus, D. Wagner, and E. Draper, "APACHE III study design: analytic plan for evaluation of severity and outcome in intensive care unit patients. Development of APACHE," Critical Care Medicine, vol. 17, pp. S181-S185, 1989.

[20] S. P. Baker, B. O’Neill, W. Haddon, and W. B. Long, “The injury severity score: a method for describing patients with multiple injuries and evaluating emergency care," Journal of Trauma, vol. 14, no. 3, pp. 187-196, 1974.

[21] G. B. S. Wells, D. O’Connell, J. Peterson, V. Welch, M. Losos, and P. Tugwell, "The Newcastle-Ottawa Scale (NOS) for assessing the quality of nonrandomized studies in metaanalyses".

[22] L. A. Sim, L. McGovern, M. B. Elamin, B. A. Swiglo, P. J. Erwin, and V. M. Montori, "Effect on bone health of estrogen preparations in premenopausal women with anorexia nervosa: a systematic review and meta-analyses," International Journal of Eating Disorders, vol. 43, no. 3, pp. 218-225, 2010.

[23] R. DerSimonian and N. Laird, "Meta-analysis in clinical trials," Controlled Clinical Trials, vol. 7, no. 3, pp. 177-188, 1986.

[24] J. P. T. Higgins, S. G. Thompson, J. J. Deeks, and D. G. Altman, "Measuring inconsistency in meta-analyses," British Medical Journal, vol. 327, no. 7414, pp. 557-560, 2003.

[25] D. G. Altman and J. M. Bland, "Statistics notes-interaction revisited: the difference between two estimates," British Medical Journal, vol. 326, no. 7382, p. 219, 2003.

[26] J. A. C. Sterne and M. Egger, "Funnel plots for detecting bias in meta-analysis: guidelines on choice of axis," Journal of Clinical Epidemiology, vol. 54, no. 10, pp. 1046-1055, 2001.

[27] F. J. Abelha, M. Botelho, V. Fernandes, and H. Barros, "Determinants of postoperative acute kidney injury," Critical Care, vol. 13, no. 3, article R79, 2009.

[28] S. M. Bagshaw, C. George, and R. Bellomo, "Early acute kidney injury and sepsis: a multicentre evaluation," Critical Care, vol. 12, no. 2, article R47, 2008.

[29] S. M. Bagshaw, C. George, R. T. N. Gibney, and R. Bellomo, "A multi-center evaluation of early acute kidney injury in critically ill trauma patients," Renal Failure, vol. 30, no. 6, pp. 581-589, 2008.

[30] I. Bahar, A. Akgul, M. A. Ozatik et al., "Acute renal failure following open heart surgery: risk factors and prognosis," Perfusion, vol. 20, no. 6, pp. 317-322, 2005.

[31] F. Barrantes, J. Tian, R. Vazquez, Y. Amoateng-Adjepong, and C. A. Manthous, "Acute kidney injury criteria predict outcomes of critically ill patients," Critical Care Medicine, vol. 36, no. 5, pp. 1397-1403, 2008.

[32] B. A. Boucher, B. C. Coffey, D. A. Kuhl, E. A. Tolley, and T. C. Fabian, "Algorithm for assessing renal dysfunction risk in critically ill trauma patients receiving aminoglycosides," American Journal of Surgery, vol. 160, no. 5, pp. 473-480, 1990.

[33] T. Bove, M. G. Calabrò, G. Landoni et al., "The incidence and risk of acute renal failure after cardiac surgery," Journal of Cardiothoracic and Vascular Anesthesia, vol. 18, no. 4, pp. 442-445, 2004.

[34] L. S. Chawla, L. Abell, R. Mazhari et al., "Identifying critically ill patients at high risk for developing acute renal failure: a pilot study," Kidney International, vol. 68, no. 5, pp. 22742280, 2005.

[35] G. Clermont, C. G. Acker, D. C. Angus, C. A. Sirio, M. R. Pinsky, and J. P. Johnson, "Renal failure in the ICU: comparison of the impact of acute renal failure and end-stage renal disease on ICU outcomes," Kidney International, vol. 62, no. 3, pp. 986-996, 2002. 
[36] P. J. Conlon, M. Stafford-Smith, W. D. White et al., "Acute renal failure following cardiac surgery," Nephrology Dialysis Transplantation, vol. 14, no. 5, pp. 1158-1162, 1999.

[37] H. L. Corwin, S. M. Sprague, G. A. DeLaria, and M. J. Norusis, "Acute renal failure associated with cardiac operations. A casecontrol study," Journal of Thoracic and Cardiovascular Surgery, vol. 98, no. 6, pp. 1107-1112, 1989.

[38] J. F. Dasta, S. L. Kane-Gill, A. J. Durtschi, D. S. Pathak, and J. A. Kellum, "Costs and outcomes of acute kidney injury (AKI) following cardiac surgery," Nephrology Dialysis Transplantation, vol. 23, no. 6, pp. 1970-1974, 2008.

[39] D. J. de Araújo Brito, V. J. S. Nina, R. V. A. H. Nina et al., "Prevalence and risk factors for acute renal failure in the postoperative of coronary artery bypass grafting," Brazilian Journal of Cardiovascular Surgery, vol. 24, no. 3, pp. 297-304, 2009.

[40] U. M. Fischer, W. K. Weissenberger, R. D. Warters, H. J. Geissler, S. J. Allen, and U. Mehlhorn, "Impact of cardiopulmonary bypass management on postcardiac surgery renal function," Perfusion, vol. 17, no. 6, pp. 401-406, 2002.

[41] E. Gomes, R. Antunes, C. Dias, R. Araújo, and A. CostaPereira, "Acute kidney injury in severe trauma assessed by RIFLE criteria: a common feature without implications on mortality?" Scandinavian Journal of Trauma, Resuscitation and Emergency Medicine, vol. 18, article 1, 2010.

[42] A. B. J. Groeneveld, D. D. Tran, J. Van der Meulen, J. J. P. Nauta, and L. G. Thijs, "Acute renal failure in the medical intensive care unit: predisposing, complicating factors and outcome," Nephron, vol. 59, no. 4, pp. 602-610, 1991.

[43] M. Hilberman, B. D. Myers, and B. J. Carrie, "Acute renal failure following cardiac surgery," Journal of Thoracic and Cardiovascular Surgery, vol. 77, no. 6, pp. 880-888, 1979.

[44] C. E. Hobson, S. Yavas, M. S. Segal et al., "Acute kidney injury is associated with increased long-term mortality after cardiothoracic surgery," Circulation, vol. 119, no. 18, pp. 24442453, 2009.

[45] G. Landoni, T. Bove, M. Crivellari et al., "Acute renal failure after isolated CABG surgery: six years of experience," Minerva Anestesiologica, vol. 73, no. 11, pp. 559-565, 2007.

[46] B. G. Loef, A. H. Epema, T. D. Smilde et al., "Immediate postoperative renal function deterioration in cardiac surgical patients predicts in-hospital mortality and long-term survival," Journal of the American Society of Nephrology, vol. 16, no. 1, pp. 195-200, 2005.

[47] M. D. N. Machado, R. C. Miranda, I. T. Takakura et al., "Acute kidney injury after on-pump coronary artery bypass graft surgery," Arquivos Brasileiros de Cardiologia, vol. 93, no. 3, pp. 230-252, 2009.

[48] C. M. Mangano, L. S. Diamondstone, J. G. Ramsay, A. Aggarwal, A. Herskowitz, and D. T. Mangano, "Renal dysfunction after myocardial revascularization: risk factors, adverse outcomes, and hospital resource utilization," Annals of Internal Medicine, vol. 128, no. 3, pp. 194-203, 1998.

[49] S. E. Mataloun, F. R. Machado, A. P. R. Senna, H. P. Guimarães, and J. L. G. Amaral, "Incidence, risk factors and prognostic factors of acute renal failure in patients admitted to an intensive care unit," Brazilian Journal of Medical and Biological Research, vol. 39, no. 10, pp. 1339-1347, 2006.

[50] C. V. Thakar, A. Christianson, R. Freyberg, P. Almenoff, and M. L. Render, "Incidence and outcomes of acute kidney injury in intensive care units: a Veterans Administration study," Critical Care Medicine, vol. 37, no. 9, pp. 2552-2558, 2009.
[51] D. D. Tran, M. A. Cuesta, and P. L. Oe, "Acute renal failure in patients with severe civilian trauma," Nephrology Dialysis Transplantation, vol. 9, supplement 4, pp. 121-125, 1994.

[52] L. A. Ward, G. N. Coritsidis, and C. P. Carvounis, "Risk factors to predict renal failure and death in the medical intensive care unit," Journal of Intensive Care Medicine, vol. 11, no. 2, pp. 114-119, 1996.

[53] R. G. Wilkins and E. B. Faragher, "Acute renal failure in an intensive care unit: incidence, prediction and outcome," Anaesthesia, vol. 38, no. 7, pp. 628-634, 1983.

[54] G. Zanardo, P. Michielon, A. Paccagnella et al., "Acute renal failure in the patient undergoing cardiac operation: prevalence, mortality rate, and main risk factors," Journal of Thoracic and Cardiovascular Surgery, vol. 107, no. 6, pp. 14891495, 1994.

[55] D. N. Cruz, Z. Ricci, and C. Ronco, "Clinical review: RIFLE and AKIN-time for reappraisal," Critical care, vol. 13, no. 3, p. 211, 2009. 


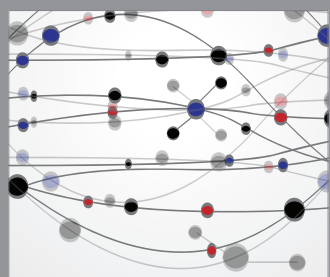

The Scientific World Journal
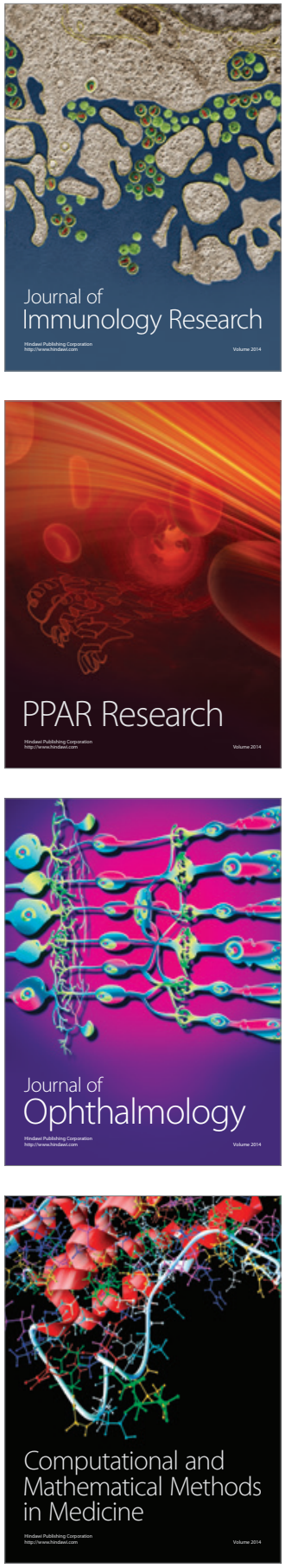

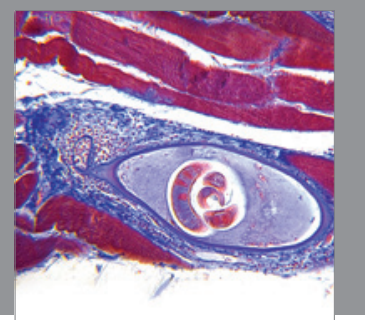

Gastroenterology

Research and Practice
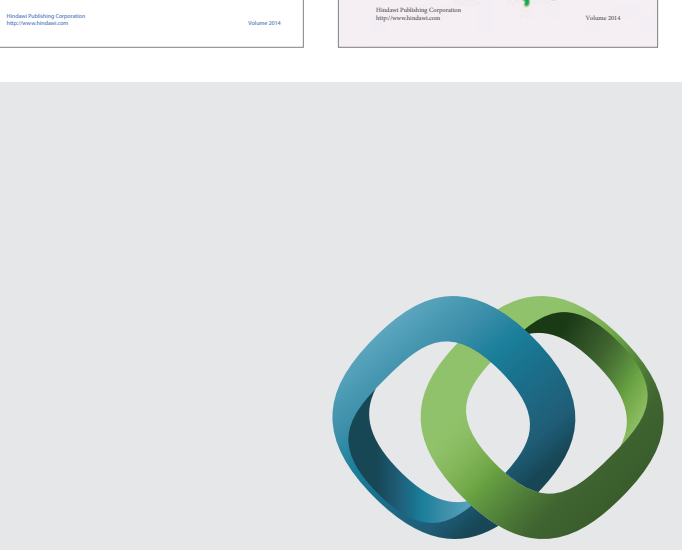

\section{Hindawi}

Submit your manuscripts at

http://www.hindawi.com
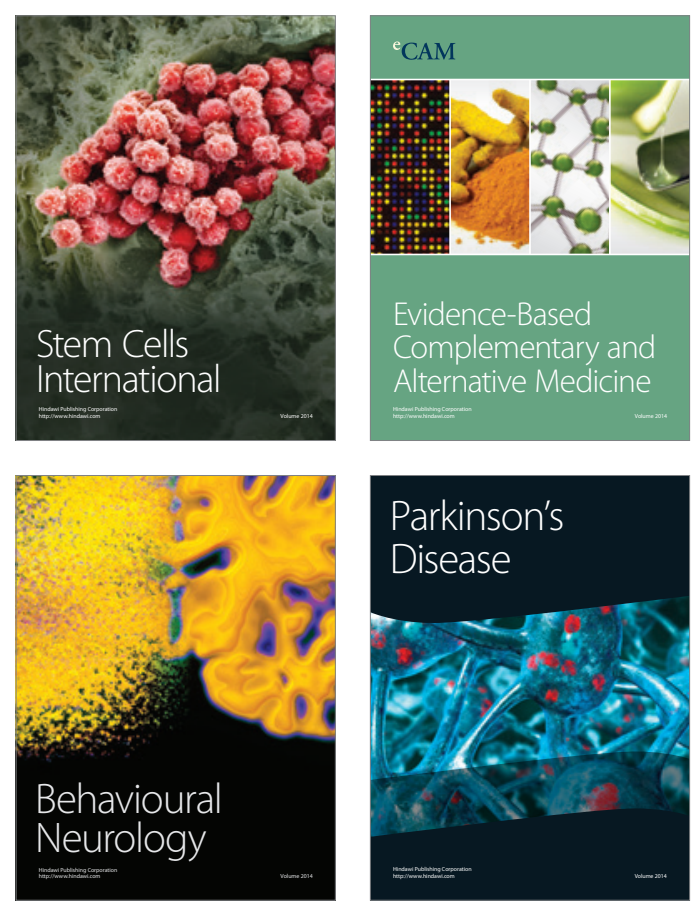

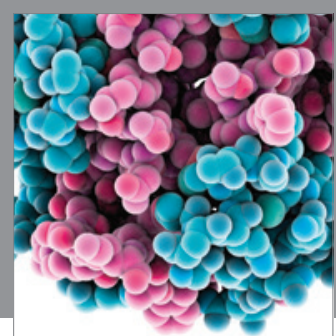

Journal of
Diabetes Research

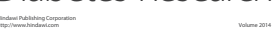

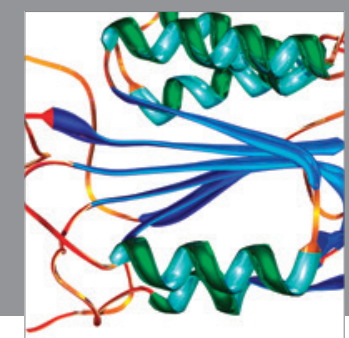

Disease Markers
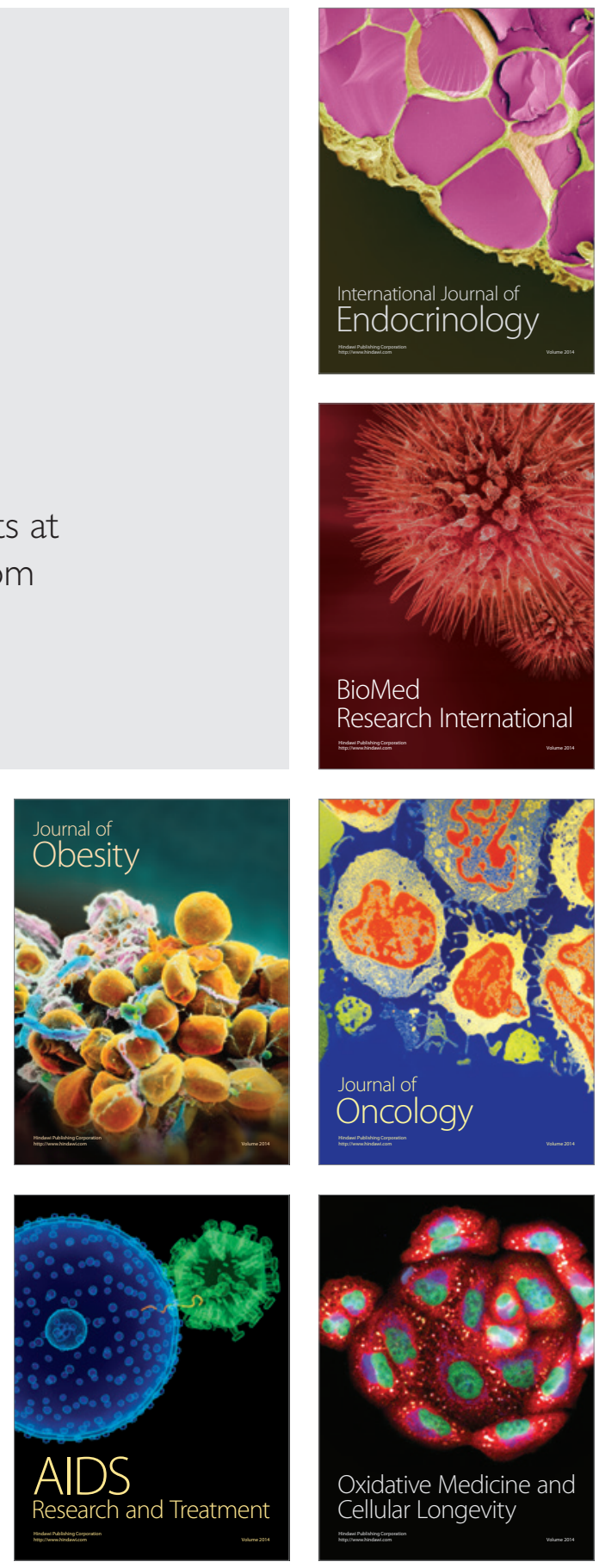